\title{
Baroclinic Kelvin Waves in a Rotating Circular Basin
}

\author{
R. N. Ibragimov*
}

Department of Mathematics, University of Texas at Brownsville, TX 78520, USA

\begin{abstract}
A linear, uniformly stratified ocean model is used to investigate propagation of baroclinic Kelvin waves in a cylindrical basin. It is found that smaller wave amplitudes are inherent to higher mode individual terms of the obtained solutions that are also evanescent away of a costal line toward the center of the circular basin. It is also shown that the individual terms if the obtained solutions can be visualized as spinning patterns in rotating stratified fluid confined in a circular basin. Moreover, the fluid patterns look rotating in an anticlockwise sense looking above the North Pole and that spinning is more intensive for smaller mode numbers. Finally, we observe the existence of the oceanic region where the pressure increases relatively rapidly with the depth.
\end{abstract}

Keywords and phrases: internal gravity waves

Mathematics Subject Classification: 35E15

\section{Introduction}

One of the main reasons for studying internal waves remains the fact that they are suspected to play an important role in the dynamics of the ocean, especially in affecting the large-scale general circulation model [11]; [18]. In this paper, we address the linear baroclinic Kelvin waves propagating along a coast having a circular shape in the presence of the Earth's rotation. The inquiry is motivated by dynamically significant Coriolis forces in modeling of large-scale oceanic and atmospheric motion with applications to meteorology, climate variability models [16], the general atmospheric circulation model [6]; [7], weather prediction (see e.g., [1];[14]) as well as a variety of applications to large-scale dispersant operations e.g. Deepwater Horizon incident studied recently in [5]

Kelvin waves affecting weather and climate are of great practical importance in the Earth's atmosphere and ocean; they represent large-scale wave motion that are a special type of Poincaré waves (or simply gravity wave) that are affected by the Earth's rotation and trapped at the equator or long lateral vertical boundaries such as coastlines or mounting ranges. The existence of the Kelvin wave relies on (i) a gravity and stable stratification for sustaining a gravitational oscillation, (ii) significant Coriolis acceleration, and (iii) the presence of vertical boundaries or the equator. There are two basic types of Kelvin waves: boundary trapped and equatorially trapped. Each type of Kelvin wave may be further subdivided into surface and internal Kelvin waves. Surface, or barotropic, waves penetrate the entire depth of the fluid. Internal Kelvin waves are often found in a layer with large density gradients; the density gradient acts as

*Corresponding author. E-mail: ranis.ibragimov@utb.edu 
an interface that allows the existence of internal gravity waves. Examples of such density gradients are the oceanic thermocline (a layer of large vertical temperature gradient separating a shallow layer of warm surface water about 50-200 m deep and a much deeper layer of cold water below) and the lower edge of an atmospheric inversion, a layer in which temperature increases with height. Like Poincaré waves, Kelvin waves can also propagate vertically in a continuously stratified geophysical fluid. Internal, or baroclinic, Kelvin waves behave in the same manner as the surface waves except that the motion varies with depth. For internal Kelvin waves, the pressure gradient force normal to the lateral boundary arises from the tilt of the interface and is balanced by the Coriolis force associated with the vertical differential flow parallel to the boundary.

Atmospheric Kelvin waves play an important role in the adjustment of the tropical atmosphere to convective latent heat release, in the stratospheric quasibiennial oscillating, and in the generation and maintance of the Madden-Julian Oscillation. Oceanic Kelvin waves play a critical role in tidal motion, in the adjustment of the tropical ocean to wind stress forcing, and in generating and sustaining the El Niño Southern Oscillaton[8].

The internal Kelvin wave speed depends on the density difference across the interface and is normally much slower than that of surface Kelvin waves. In the ocean, the typical speed for internal costal Kelvin waves is of order of $1 \mathrm{~m} \mathrm{~s}^{-1}$ and the Rossby radius of deformation is of the order of $10 \mathrm{~km}$ in the latitudes. Evidence for costal Kelvin wave propagation along the eastern boundary of the Pacific has been observed in costal sea level and temperature records. Internal costal Kelvin waves can be generated by windinduced, time-dependent costal upwelling. Costal upwelling (downelling) is cased by an Ekman mass flux transported offshore (onshore) and forced by longshore winds. The disturbances can then propagate along the coast as boundary-trapped internal Kelvin waves.

\section{Linear cylindrical internal wave field}

In this paper, we address the equations of motion for internal waves affected by the earth's rotation. The axes are $x$ (assumed eastward for definiteness) $y$ (northward) and $\widehat{k}$ is the unit vector with $z$ in the vertical direction (opposite gravity). The fluid velocity is $\vec{u}=(u, v, w)$ relative to the Cartesian coordinate system $(x, y, z)$. The hydrostatic approximation is assumed and the equations of motion are linearized about a background stable density profile $\bar{\rho}(z)$ with the associated buoyancy frequency. Motion is considered to be outside frictional boundary layers, allowing viscosity and diffusion to be neglected. In the theory of ocean circulation and within meteorological applications, it is commonplace to make the Boussinesq approximation in which the full variation of density is retained only in the buoyancy force in the vertical momentum balance. Wherever else it occurs, in the horizontal momentum balance, and in the continuity equation, density is replaced by a constant (in the simplest implementation). In particular, this means that the three-dimensional velocity field is assumed to be solenoidal (see e.g. [17]). Within the Boussinesq approximation, the governing equations of motion for internal waves, observed in a system of coordinates rotating with angular velocity $\vec{\Omega}$ are written as a non-hydrostatic analytical model in the form (see e.g., [3])

$$
\begin{aligned}
\rho_{0}\left[\frac{\partial \vec{u}}{\partial t}+\vec{u} \cdot \nabla \vec{u}+2 \vec{\Omega} \times \vec{u}\right] & =-\nabla p-g \rho \widehat{k}, \\
\frac{\partial \rho}{\partial t}+\vec{u} \cdot \nabla \rho+w \frac{d \bar{\rho}}{d z} & =0, \\
\nabla \cdot \vec{u} & =0,
\end{aligned}
$$

where $g$ is the acceleration due to gravity so that $p$ and $\rho$ are to be interpreted as the pressure and density departures from their mean state

$$
\bar{\rho}(z)=-\frac{\rho_{0}}{g} N^{2} z, \quad \bar{p}(z)=p_{0}-\rho_{0} g z-g \int_{0}^{z} \bar{\rho}(\xi) d \xi,
$$


in which $\rho_{0}$ is the constant reference density, $N$ is the buoyancy frequency defined by

$$
N^{2}=-\frac{g}{\rho_{0}} \frac{d \bar{\rho}}{d z}
$$

and we require $\rho_{0}+\bar{\rho}$ and $\bar{p}$ to be consistent with the state of rest, i.e.,

$$
\frac{d \bar{p}}{d z}=-\left(\rho_{0}+\bar{\rho}\right) g
$$

The traditional $f$-plane approximation is made whereby we take $2 \vec{\Omega}=(0,0, f)$, where $f$ is the inertial frequency which depends on the rotation rate of the earth (angular velocity $\Omega=2 \pi \mathrm{rad} /$ day $\approx 0.73 \times 10^{-4}$ $\mathrm{s}^{-1}$ ). The quantity $N$ measures the degree of density stratification of a fluid with average potential density $\rho(z)$ and thus represents the frequency with which a vertically displaced fluid element would be expected to oscillate because of restoring buoyancy forces. If the displacement is not strictly vertical, as in the case for internal waves, the restoring force is less so the frequency of oscillations is reduced.

Let there be a cylindrical model basin with radius $r_{0}$ and of the depth $H$ with $z \in[0, H]$. We employ the usual cylindrical coordinates $(r, \theta, z)$ via $x=r \cos \theta, y=r \sin \theta, z=z$ so that the rate-of-strain tensor is

$$
e_{r r}=\frac{\partial u_{r}}{\partial r}, \quad e_{\theta \theta}=\frac{1}{r} \frac{\partial u_{\theta}}{\partial \theta}, \quad e_{r \theta}=\frac{r}{2} \frac{\partial}{\partial r}\left(\frac{u_{\theta}}{r}\right)+\frac{1}{2 r} \frac{\partial u_{r}}{\partial \theta} .
$$

Then the linearized Boussinesq model (2.1) - (2.3) is written as

$$
\begin{aligned}
\frac{\partial u_{r}}{\partial t}-f u_{\theta} & =-\frac{\partial p}{\partial r} \\
\frac{\partial u_{\theta}}{\partial t}+f u_{r} & =-\frac{1}{r} \frac{\partial p}{\partial \theta}, \\
\frac{\partial w}{\partial t} & =-\frac{\partial p}{\partial z}-\rho g, \\
\frac{\partial \rho}{\partial t} & =\frac{N^{2}}{g} w \\
\frac{1}{r} \frac{\partial\left(r u_{r}\right)}{\partial r}+\frac{1}{r} \frac{\partial u_{\theta}}{\partial \theta}+\frac{\partial w}{\partial z} & =0
\end{aligned}
$$

Our model is idealized by assuming $N$ to be uniform over the extent of the fluid. This corresponds to a vertically linear density variation. While this simplification is commonly used in laboratory and theoretical studies and it is quite reasonable for the thermocline region, it is not common in the deep region of the ocean with the except when considering short wavelengths in comparisons with the scale of density changes [15]. At low frequencies, close to $f$, rotational effects are important. Such internal waves are sometimes called inertial-internal waves. At high frequencies, close to $N$ and far from $f$, rotational effects are negligible.

\section{Fundamental solution}

Elimination of the pressure terms from (2.7), (2.8) and expressing $\partial w / \partial z$ from (2.10) yields

$$
\frac{\partial \varphi}{\partial t}-\frac{f r g}{N^{2}} \frac{\partial^{2} \rho}{\partial t \partial z}=0
$$

where the vorticity $\varphi$ is defined as

$$
\varphi=\frac{\partial\left(r u_{\theta}\right)}{\partial r}-\frac{\partial u_{r}}{\partial \theta}
$$

We next define

$$
F=\varphi-\frac{f r g}{N^{2}} \frac{\partial \rho}{\partial z}
$$


As follows from (3.1), $F$ can be regarded as time independent, i.e., $F=F(r, \theta, z)$. For steady geostrophic flows, $F \neq 0$ (see e.g. [13]). However, since we are looking for solutions periodic in time with zero mean, in the frame of the present analysis, we set $F=0$. Taking the latter assumption into account, we recast the model (2.7) - (2.11) as a single equation for the pressure perturbation only which is much used in dynamical meteorology (see e.g. [4]). The resulting equation is

$$
\Delta p_{t t}+N^{2} \Delta_{h} p+f^{2} p_{z z}=0
$$

where subscripts denote partial differentiation,

$$
\Delta_{h}=\frac{1}{r} \frac{\partial}{\partial r}\left(r \frac{\partial}{\partial r}\right)+\frac{1}{r^{2}} \frac{\partial^{2}}{\partial \theta^{2}}
$$

is the two-dimensional Laplace-Beltrami operator in polar angles and

$$
\Delta=\Delta_{h}+\frac{\partial^{2}}{\partial z^{2}} .
$$

We employ the usual normal mode analysis (e.g., [3] or [12]) and allow solutions to be found as expansions of vertical modes $\psi_{n}(z)$ of the form

$$
p=\sum_{n} p_{n}(r, \theta, t) \psi_{n}(z)
$$

where the uniform mean vertical stratification $\bar{\rho}(z)$ is absorbed into the definition of the pressure and density so that $p$ has units of (potential) energy per unit mass whereas e.g. $\rho$ represents (dimensionless) density anomaly [12].

Following [4], we write the bottom boundary conditions at $z=0$ and the rigid lid approximation for the free surface boundary condition at $z=H$ as

$$
p_{z}(r, \theta, 0, t)=p_{z}(r, \theta, H, t)=0 .
$$

In view of the boundary condition (3.8), it is more convenient to look for a specific example of the idealized vertical profile $\psi_{n}(z)$, e.g.

$$
p=\sum_{n} \widehat{p}_{n}(r) \cos \left(n \theta-\omega_{n} t\right) \cos \left(m_{n} z\right),
$$

where $m_{n}$ is the vertical wavenumber vector.

One can readily verify that the first boundary condition (3.8) is automatically satisfied with the choice of the sinusoidal vertical structure (3.9) whereas the second boundary condition (3.8) implies that there exist an infinite number of internal wave modes satisfying the dispersion relationship for internal waves, i.e.

$$
\omega_{n}^{2}=\frac{\alpha_{n}^{2} N^{2}+m_{n}^{2} f^{2}}{m_{n}^{2}+\alpha_{n}^{2}}, \quad m_{n} H=n \pi, \quad n= \pm 1, \pm 2, . .
$$

Here $\alpha_{n}$ is a constant having the dimension of the azimuthal wavenumber vector.

Substitution of the presentation (3.9) into Eq. (3.4) yields a linear ODE

$$
\frac{d^{2} \widehat{p}_{n}}{d r^{2}}+\frac{1}{r} \frac{d \widehat{p}_{n}}{d r}+\left[\frac{m_{n}^{2}\left(\omega_{n}^{2}-f^{2}\right)}{N^{2}-\omega_{n}^{2}}-\frac{n^{2}}{r^{2}}\right] \widehat{p}_{n}=0 .
$$

In the mid/high latitude upper ocean, $N$ is typically one or two orders of magnitude larger than $f$ $\left(N \sim 10^{-3}\right.$ to $\left.10^{-2} \mathrm{~s}^{-1} ; f \sim 10^{-4} \mathrm{~s}^{-1}\right)$ and so hereafter we limit our analysis to the case

$$
|f|<\left|\omega_{n}\right|<|N|
$$




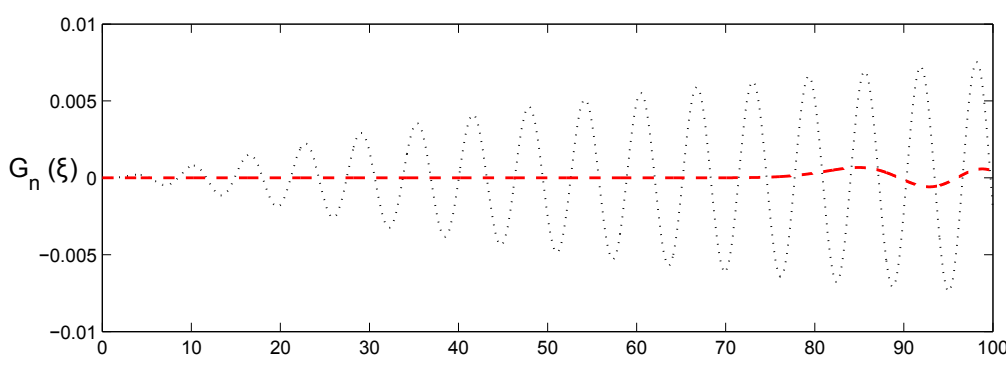

(a)

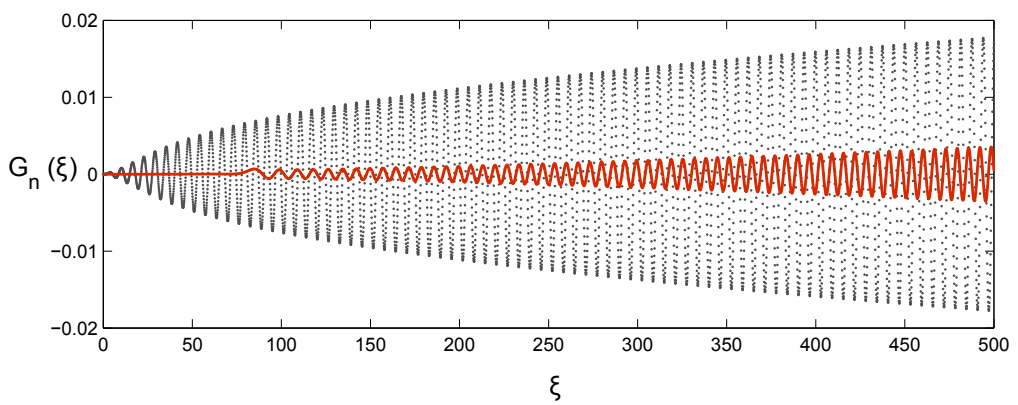

(b)

Figure 1. The coastal boundary condition $G_{n}=0$ for $n=1$ (dashed grey line) and $n=10$ (dashed red line) at the fixed parameters $N=10^{-3} \mathrm{~s}^{-1}$ and $f=0.5 \times 10^{-4} \mathrm{~s}^{-1}$ which is a typical value of at $20^{\circ}$ North. For comparison purposes, panel (a) shows the case when $\xi \in\left[\varepsilon, \xi_{\max }\right]$ for $\xi_{\max }=100$ whereas the plot of $G_{n}$ for $\xi_{\max }=500$ is shown in panel (b). In both cases, we set $\varepsilon=0.01$.

Then, as follows from (3.10),

$$
\frac{m_{n}^{2}\left(\omega_{n}^{2}-f^{2}\right)}{N^{2}-\omega_{n}^{2}}=\alpha_{n}^{2}>0 .
$$

We next introduce a new independent variable $\xi$ defined via $\xi=\alpha_{n} r$ and regard $\widehat{p}$ as a function of $\xi$. Thus Eq. (3.11) is written as the Bessel's equation of order $n$ with the single singular point at $\xi=0$ [19].

$$
\xi^{2} \frac{d^{2} \widehat{p}_{n}}{d \xi^{2}}+\xi \frac{d \widehat{p}_{n}}{d \xi}+\left(\xi^{2}-n^{2}\right) \widehat{p}_{n}=0 .
$$

Correspondingly, the solution of Eq. (3.14) is given by the Bessel function of the first kind of order $n$ :

$$
\widehat{p}_{n}(\xi)=J_{n}(\xi)=\left(\frac{\xi}{2}\right)^{n} \sum_{k=0}^{\infty} \frac{(-1)^{k}}{k !(k+n) !}\left(\frac{\xi}{2}\right)^{2 k} .
$$

Thus, away of the coastal boundary $r=r_{0}$, the solution of Eq. (3.11) is written as

$$
p(r, \theta, z, t)=\sum_{n} J_{n}\left(\alpha_{n} r\right) \cos \left(n \theta-\omega_{n} t\right) \cos \left(m_{n} z\right) .
$$

\subsection{Kelvin waves}

To take into account the presence of a coastal line located at $r=r_{0}$, we rewrite the equations (2.7) and (2.8) as the following single partial differential equation

$$
\left(\frac{\partial^{2}}{\partial t^{2}}+f^{2}\right) u_{r}=-\frac{f}{r} \frac{\partial p}{\partial \theta}-\frac{\partial^{2} p}{\partial t \partial r} .
$$


In order to model the Kelvin waves, we employ the boundary condition of no normal flow at the coast. Substituting the solution (3.16) into the equation (3.17) and evaluating at $r=r_{0}$, we obtain the additional boundary condition

$$
G_{n}:=\frac{d}{d \xi} \ln \left[J_{n}(\xi)\right]-\frac{f n}{\omega_{n} \xi}=0 \quad \text { at } \quad \xi=\xi_{0},
$$

where we denote $\xi_{0}=\alpha_{n} r_{0}$. The boundary condition (3.18) serves as an additional condition for the eignefrequencies $\omega_{n}$ obeying the condition (3.12).

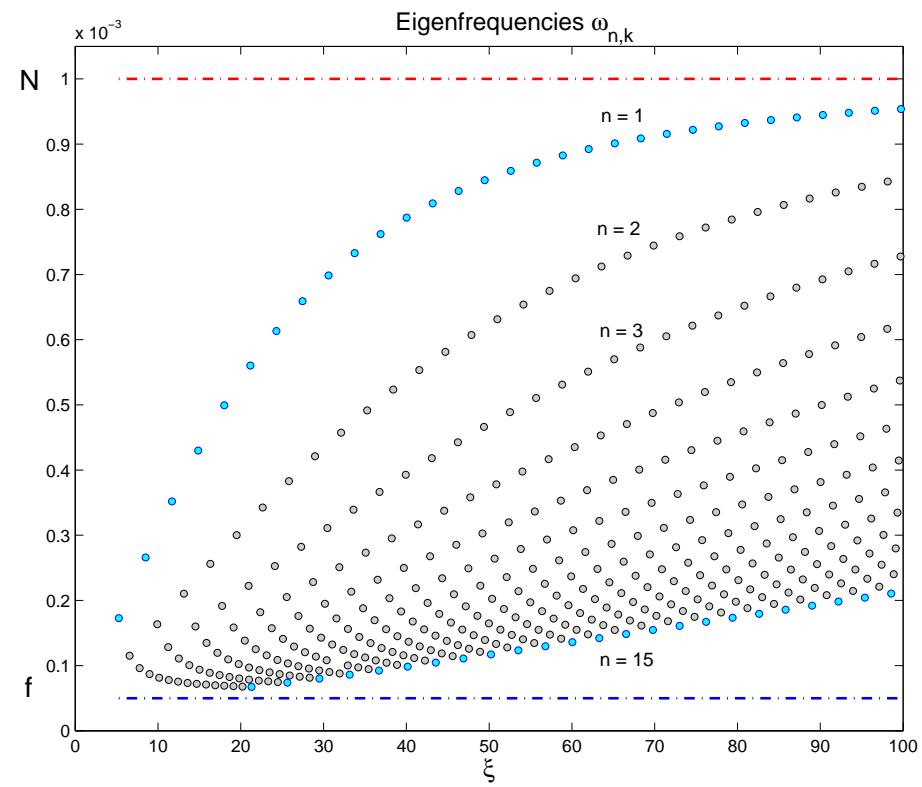

Figure 2. Distribution of the first few eigenfrequencies $\omega_{n, k}$ for $n \in[1,15]$ and $k \in[1,30]$ at latitude $\phi=20^{\circ}$ North. The domain of the existing eigenfrequencies is embraced by the Coriolis parameter $f$ and the buoyancy frequency $N$ so that the eigenfrequencies band of internal Kelvin waves is limited, $|f|<\left|\omega_{n, k}\right|<|N|$.

The eigenfrequencies $\omega_{n}$ are determined as follows: First, we find graphically the roots $\xi_{k}$ $(k=1,2,3, \ldots)$ of the equation $G_{n}=0$ given by $(3.18)$. As an illustrative example, the further simulations were done using the values of parameters as given in the table below:

\begin{tabular}{|c|c|c|c|}
\hline Parameter & Value & Dimensions & Description \\
\hline$\phi$ & $\theta=20^{\circ}$ North & deg & Latitude \\
\hline$N$ & $10^{-3}$ & $1 / \mathrm{s}$ & Buoyancy frequency \\
\hline$f$ & $0.5 \times 10^{-4} \mathrm{~s}^{-1}$ & $1 / \mathrm{s}$ & Coriolis parameter \\
\hline$n$ & $n \in\left[n_{\min }, n_{\max }\right]$ & - & Mode number band \\
\hline$\left|n_{\max }\right|$ & 15 & - & Maximum mode number \\
\hline$\left|n_{\min }\right|$ & 1 & - & Minimum mode number \\
\hline$H$ & 1000 & $\mathrm{~m}$ & Basin depth \\
\hline$r_{0}$ & 10000 & $\mathrm{~m}$ & Radius of the basin \\
\hline
\end{tabular}

(Table 1)

As a particular example, Figure 1 is used to show the zeros of $G_{n}$ for $n=1$ and $n=10$ with $\xi \in\left[\varepsilon, \xi_{\max }\right]$ in which $\xi_{\max }=100$ (panel a) and $\xi_{\max }=500$ (panel b). In the both cases, we set $\varepsilon=0.01$. We remark 


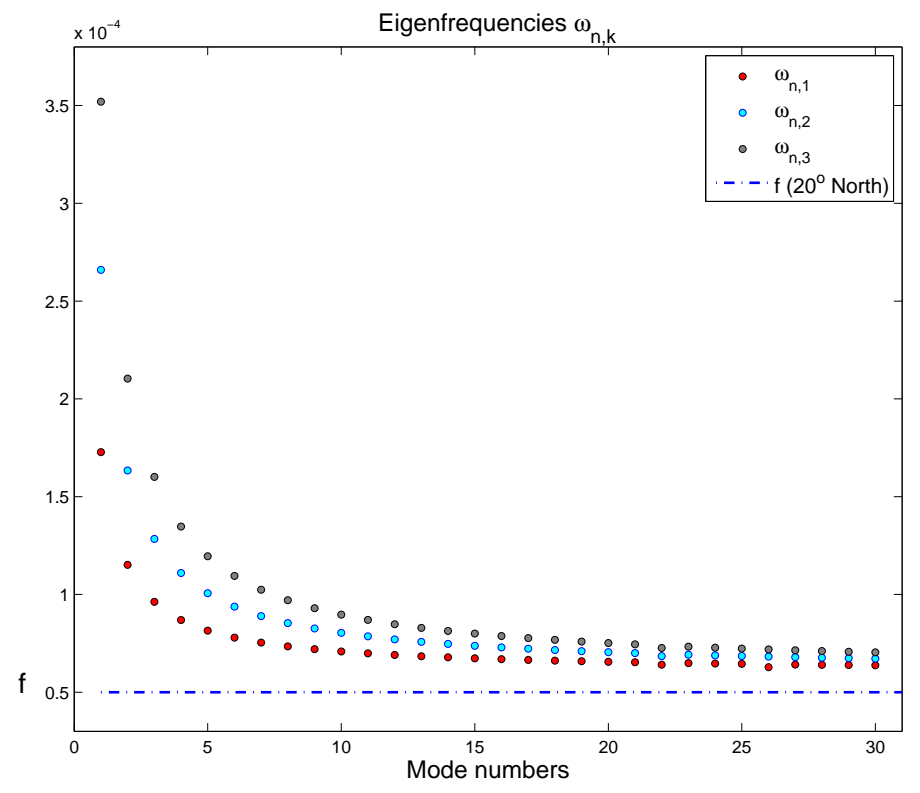

FiguRE 3. First three eignefrequencies $\omega_{n, k}$ versus mode numbers for $n \in[1,30]$ and $k \in[1,3]$ at latitude $\phi=20^{\circ}$ North.

that the numerical values of roots for $G_{n}=0$ do not depend on the particular choice of the basin's depth $H$ and the radius $r_{0}$ but only depend on the aspect ratio $\gamma=H / r_{0}$. Particularly, in our simulations, according to Table 1 , we further set $\gamma=0.1$.

Next, for each found root $\xi_{k}$, the corresponding eigenfrequencies $\omega_{n, k}$ are found from the dispersion relation (3.10). As a particular example, Figure 2 shows the distribution of the first few eigenfrequencies $\omega_{n, k}$ versus $\xi$ for $n \in[1,15]$ and $k \in[1,30]$ at latitude $\phi=20^{\circ}$ North. The range of the obtained eigenfrequencies $\omega_{n, k}$ associated with the obtained values of $\xi_{k}$ is embedded by the Coriolis parameter $f$ and the buoyancy frequency $N$, i.e. $|f|<\left|\omega_{n, k}\right|<|N|$, corresponding to the limiting cases (horizontal and vertical) of internal waves propagation. Figure 3 is used to show the first three eigenfrequencies $\omega_{n, k}(k=1,2,3)$ versus the first 30 mode numbers. We observe that $\lim _{n \rightarrow \infty} \omega_{n, k}=f$ which agrees with conclusion on higher harmonic internal waves merging when $\omega_{n, k}=f$ along the half cones represented by possible internal wave group velocity vectors in terms of e.g. modeling of equatorial wave beams (see e.g. [9]; [10]). All further simulations were carried out using the value of latitude $\phi=20^{\circ}$ North.

\section{Analysis of solution}

Figure 4 is used to show the initial eigen-mode decomposition of the eigenfunctions

$$
p_{n, k}=\widehat{p}_{n, k}(r) \cos \left(n \theta-\omega_{n, k} t\right) \cos \left(m_{n} z\right)
$$

versus $\xi$ with the fixed values of parameters $z=333 \mathrm{~m}, \theta=\pi / 3$ and $n=1,5$ and 10. Panel (a) shows the solution (4.1) corresponding to the first solution of (3.18), i.e. $k=1$. We observe that the higher-mode solutions have smaller amplitudes and are evanescent away of the coastal line $\xi=\xi_{0}$, for which, we also remark, the value of $\xi_{0}$ depends on $\alpha_{n}$. Similar arguments extend to higher values of $k$, as illustrated on panel (b) for $k=25$. 
Figure 5 shows snapshots of the eigen-mode decomposition (4.1) for the same values of parameters as used in Figure 4 at initial time $N_{t}=0$ and a latter time of $N_{t}=100$ inertial periods, where $\phi$-dependent inertial period is defined by $2 \pi / f$ and the number of inertial periods is denoted by $N_{t}$.

The snapshots of the eigenfunctions $p_{k}$ given by (3.16), i.e.

$$
p_{k}(r, \theta, z, t)=\sum_{n=1}^{n_{\max }} \widehat{p}_{n, k}(r) \cos \left(n \theta-\omega_{n, k} t\right) \cos \left(m_{n} z\right) .
$$

corresponding to $k=25$ versus $r$ with the fixed values of parameters $z=333 \mathrm{~m}, \theta=\pi / 3$ is presented in Figure 6. The initial pressure distribution for $N_{t}=0$ is plotted by red line, a latter time $N_{t}=1$ is plotted by blue line and the intermediate times $N_{t} \in(0,1)$ are plotted by grey dashed lines.

Figure 7 is used to show the snapshots of the eigen-mode decomposition of $p_{n, k}$ given by (4.1) corresponding to $k=1$ versus the vertical distance $z$ at the fixed values of parameter $\theta=\pi / 3, r=5000 \mathrm{~m}$ and the values of inertial periods $N_{t}=[0,0.04]$ for the mode numbers $n=1$ (panel a), $n=2$ (panel b), $n=5$ (panel c) and $n=10$ (panel d). The initial time $\tau=0$ is plotted by red line and the latter time $\tau=0.04$ inertial periods is plotted by blue line.
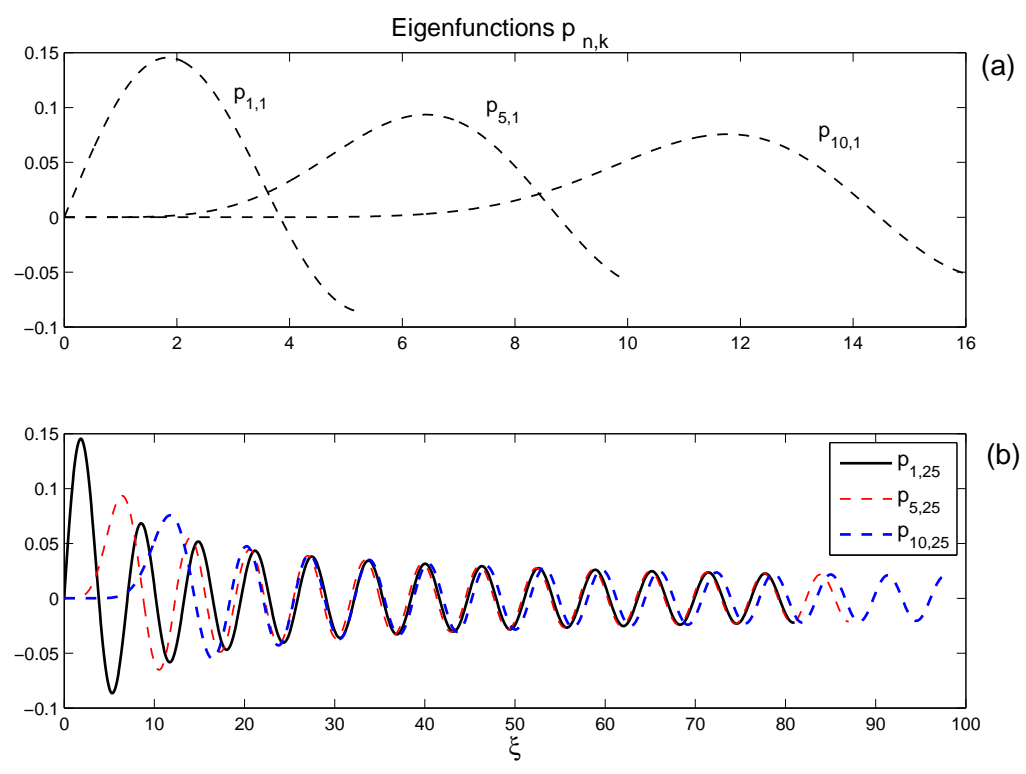

FiguRE 4. Initial distribution of the eigen-mode decomposition for the eigenfunctions $p_{n, k}$ with the fixed values of parameters $z=333 \mathrm{~m}, \theta=\pi / 3$ and $n=1,5$ and 10. The solutions correspond to $k=1$ (panel a) and $k=25$ (panel b).

Figure 8 illustrates the time series of the eigenfunctions $p_{k}$ given by (4.2) corresponding to $k=1$ versus the vertical distance $z$ at the fixed values of parameters $\theta=\pi / 3, r=5000 \mathrm{~m}$ and the values of inertial periods $N_{t}=[0,0.04]$. The initial pressure distribution for $N_{t}=0$ is plotted by red line, a latter time $N_{t}=0.04$ is plotted by blue line and the intermediate times $N_{t} \in(0,0.04)$ are plotted by grey dashed lines. We observe a region corresponding to about $700 \mathrm{~m}$ deep coastal ocean where the pressure increases relatively rapidly with depth.

Figures 9 and 10 are used to demonstrate and compare the spinning phenomena for the time series of the of the eigen-mode decomposition (4.1) for $p_{n, k}$ in $(r, \theta)-$ plane with $k=1$ and at the fixed 

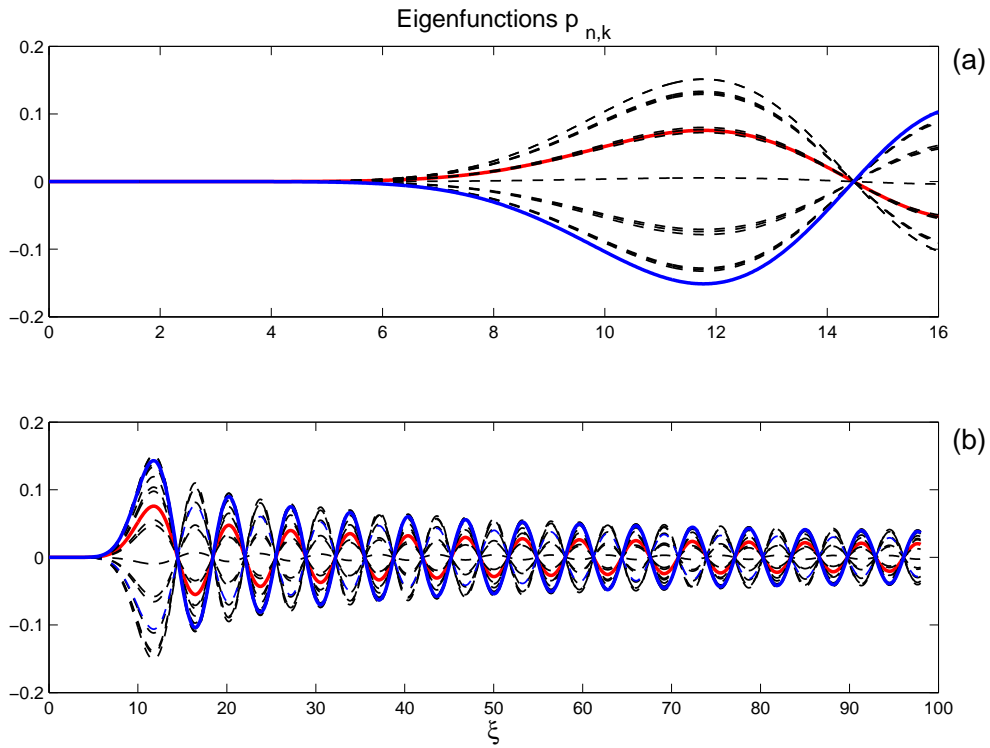

(b)

FiguRE 5. Time series for the eigenfunctions $p_{n, k}$ with the fixed values of parameters $z=333 \mathrm{~m}, \theta=\pi / 3$ and the values of time $\tau=[0,100]$ for the mode number $n=10$. The initial time $\tau=0$ is plotted by red line and the latter time of 100 inertial periods is plotted by blue line. The solutions correspond to $k=1$ (panel a) and $k=25$ (panel b).

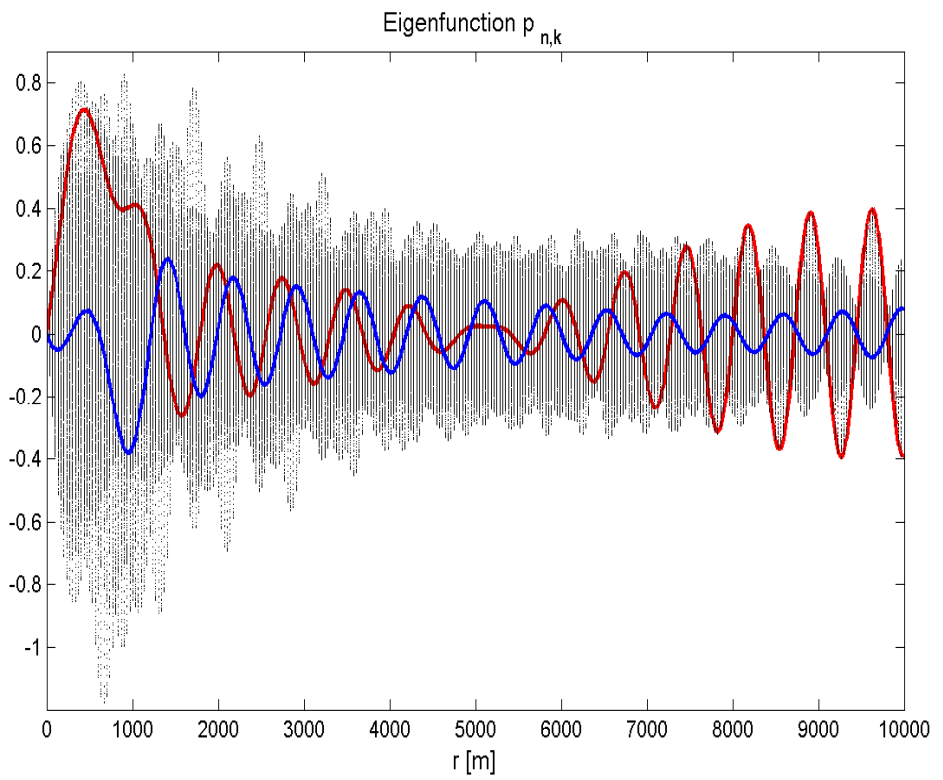

Figure 6 . Time series of the eigenfunctions $p_{k}$ given by (4.2) corresponding to $k=25$ versus $r$ with the fixed values of parameters $z=333 \mathrm{~m}, \theta=\pi / 3$.

value of $z=333 \mathrm{~m}$ for different mode numbers. Particularly, the contour plot 9 for the time series of the equipotential curves for $p_{1,1}$ reveals the counterclockwise rotation from the equilibrium position 


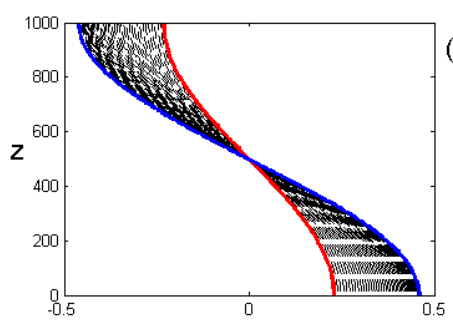

(a)

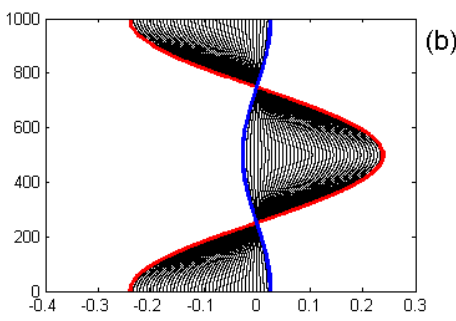

(b)
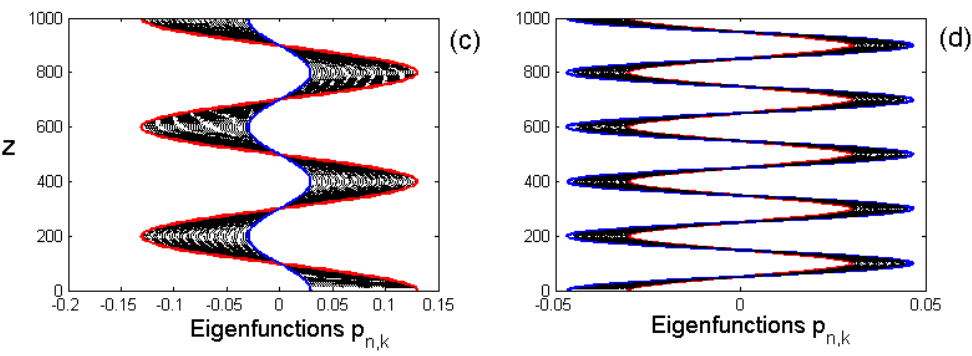

FiguRE 7 . Time series for the eigen-mode decomposition of $p_{n, k}$ corresponding to $k=1$ versus the vertical distance $z$ at the fixed values of parameter $\theta=\pi / 3, r=5000 \mathrm{~m}$ and the values of inertial periods $N_{t}=[0,0.04]$ for the mode numbers $n=1$ (panel a), $n=2($ panel b), $n=5($ panel c) and $n=10($ panel d).

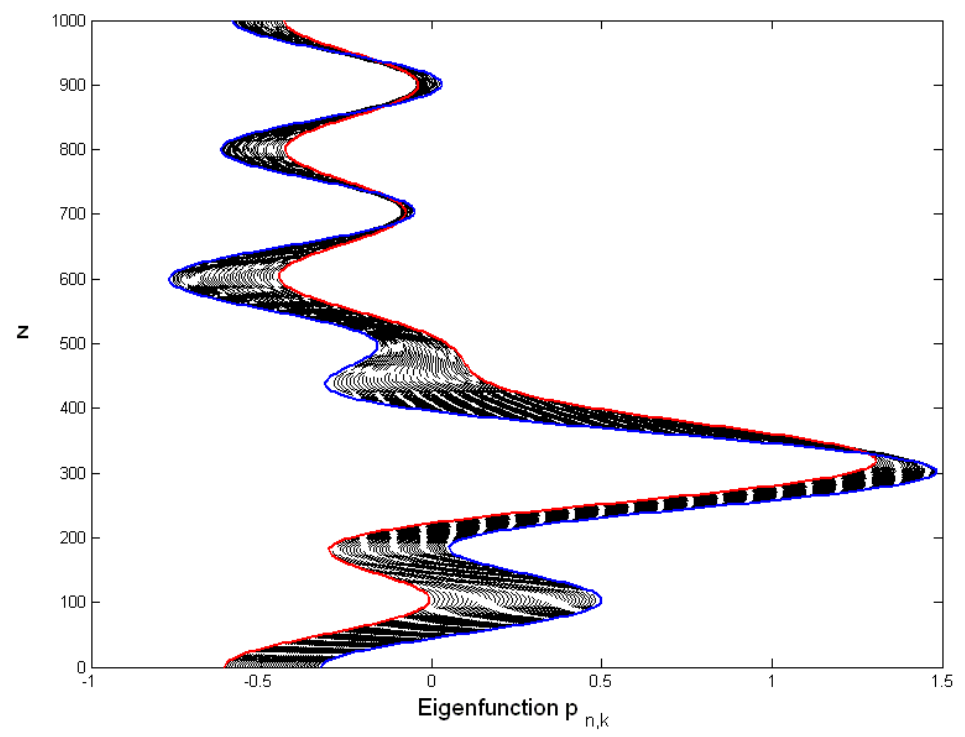

FiguRE 8 . Time series of the eigenfunctions $p_{k}$ given by (4.2) corresponding to $k=1$ versus the vertical distance $z$ at the fixed values of parameters $\theta=\pi / 3, r=5000 \mathrm{~m}$ and the values of inertial periods $N_{t}=[0,0.04]$. 

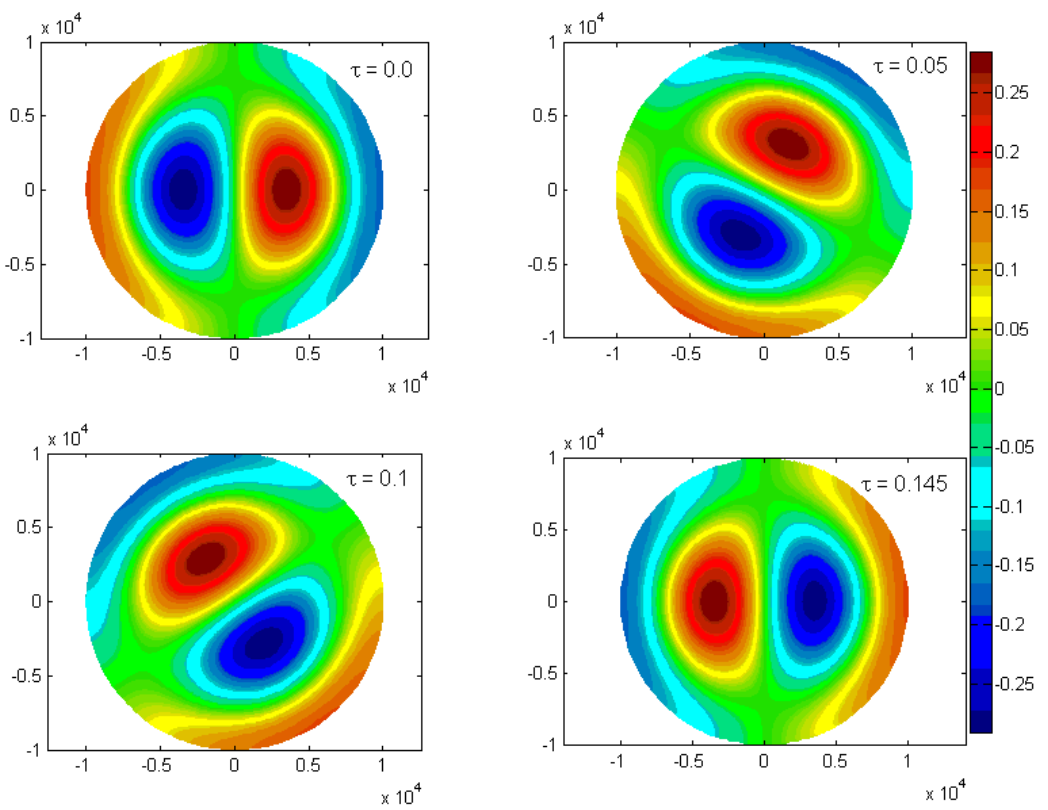

Figure 9. Time series of the eigen-mode decomposition (4.1) for $p_{1,1}$ in $(r, \theta)$-plane at the fixed value of $z=333 \mathrm{~m}$.
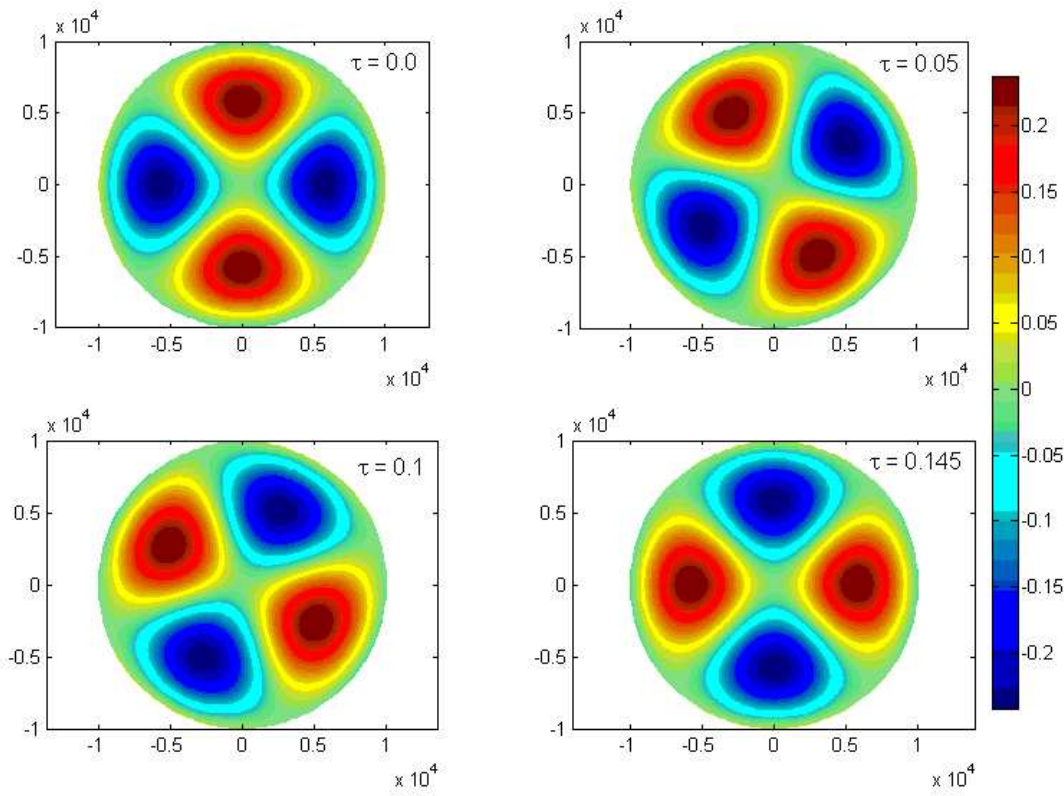

Figure 10. Time series of the eigen-mode decomposition (4.1) for $p_{2,1}$ in $(r, \theta)$-plane at the fixed value of $z=333 \mathrm{~m}$.

defined by $N_{t}=0$ at initial time to the "left" maximum azimuth deviation (half-period) attained at later 

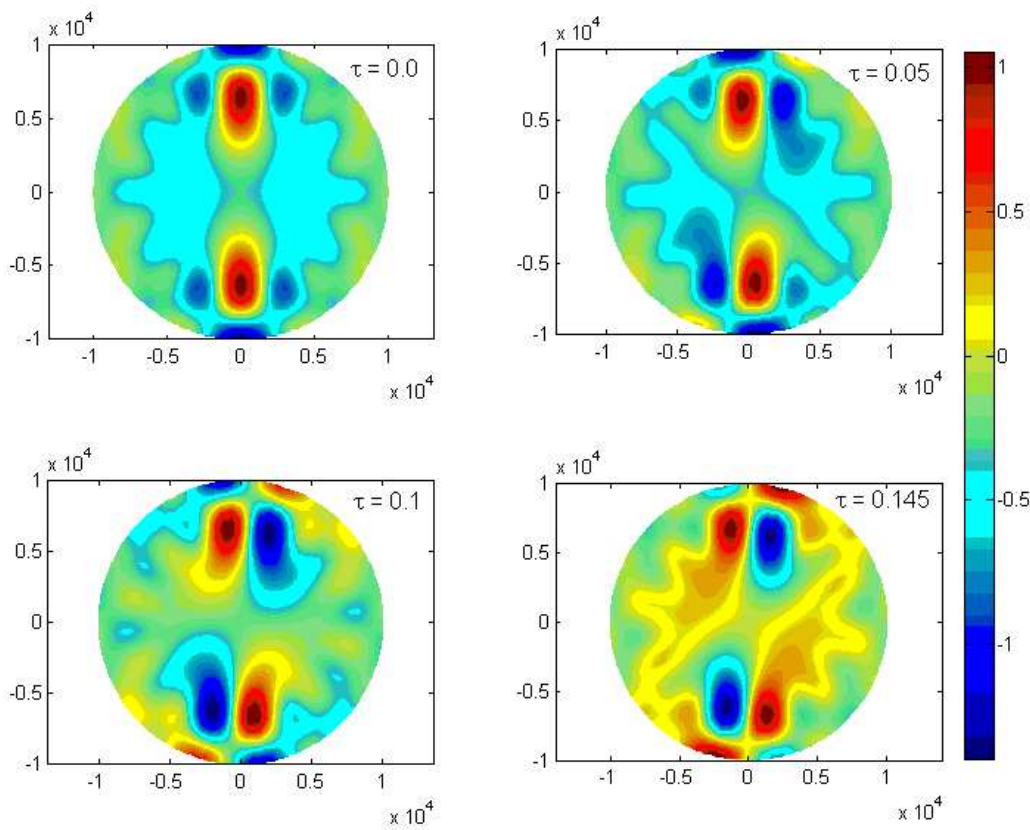

Figure 11. Time series of the eigenfunction $p_{k}$ given by $(4.2)$ in $(r, \theta)$-plane corresponding to $k=1$ at the fixed value of $z=333 \mathrm{~m}$.

time of $N_{t}=0.145$ inertial periods. Similar quantitative argument extends to higher mode numbers, as illustrated in Figure 10 for $n=2$. However, based on the comparison between the results presented in Figures 9 and 10, we observe that the spinning is less intensive for higher mode solutions.

Figure 11 shows the time series of the eigenfunction $p_{k}$ given by $(4.2)$ for $N_{t} \in(0,0.04)$ in $(r, \theta)-$ plane corresponding to $k=1$ at the fixed value of $z=333 \mathrm{~m}$.

Finally, the effects of the ocean's depth in the circular basin on the pressure distribution is illustrated in Figure 2.10 showing the time series of the eigenfunction $p_{k}$ given by $(4.2)$ in $(r, \theta)$ - plane corresponding to $k=1$ at different depths of the circular basin. The first row corresponds to the $750 \mathrm{~m}$ deep ocean whereas the rows II and III correspond to $333 \mathrm{~m}$ and $10 \mathrm{~m}$ deep respectively.

\section{Conclusion}

A simple modeling scenario in this paper was designed to examine linear baroclinic Kelvin waves propagating along a coast having a circular shape in the presence of the Earth's rotation. It is found that smaller wave amplitudes are inherent to higher mode individual terms of the obtained solutions that are also evanescent away of a costal line toward the center of the circular basin. It is also shown that the individual terms if the obtained solutions can be visualized as spinning patterns in rotating stratified fluid confined in a circular basin. Moreover, the fluid patterns look rotating in an anticlockwise sense looking above the North Pole and that spinning is more intensive for smaller mode numbers. Finally, we observe the existence of the oceanic region where the pressure increases relatively rapidly with the depth.

Our model is idealized by assuming $N$ to be uniform over the extent of the fluid. This corresponds to a vertically linear density variation. While this simplification is commonly used in laboratory and theoretical studies and it is quite reasonable for the thermocline region, it is not common in the deep 
(1)

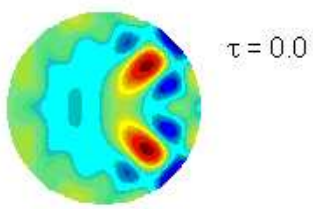

(II)

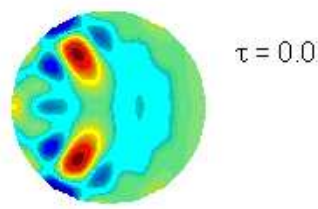

(III)

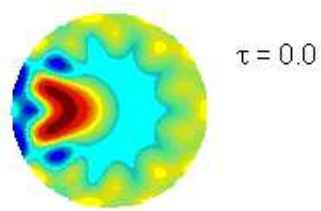

$\tau=10.0$

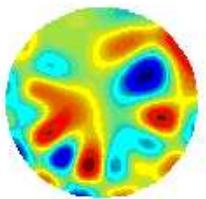

$\tau=10.0$

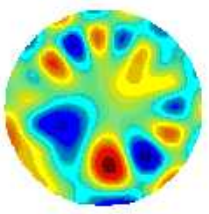

$\tau=10.0$

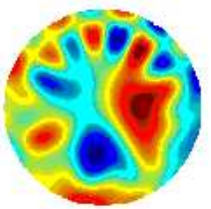

Figure 12. Time series of the eigenfunction $p_{k}$ given by $(4.2)$ in $(r, \theta)$-plane corresponding to $k=1$ at different values of $z$. The first row corresponds to the $750 \mathrm{~m}$ deep ocean whereas the rows II and III correspond to $333 \mathrm{~m}$ and $10 \mathrm{~m}$ deep respectively.

region of the ocean with the exception when considering short wavelengths in comparison with the scale of density changes [15]. This compromises the quantitative application of our results to the real ocean.

An interesting topics for further study could include nonlinear terms in the governing equations and influence of variable rotation and variable depth on baroclinic Kelvin waves.

\section{References}

[1] S. Balasuriya. Vanishing viscosity in the barotropic $\beta$-plane. J. Math.Anal. Appl. , (1997), 214, 128-150.

[2] E. Dewan, R. Picard, R. O'Neil, H. Gardiner, J. Gibson. MSX satellite observations of thunderstorm-generated gravity waves in mid-wave infrared images of the upper stratosphere. Geophys. Res. Lett., (1998), 25, 939-942.

[3] A. Gill. Atmosphere-Ocean Dynamics. New York, etc., Academic Press, 1983.

[4] G. Haltiner, R. Williams. Numerical prediction and dynamic meteorology, 1980.

[5] P. Hsieh. Application of modflow for oil reservoir simulation during the Deepwater Horizon crisis. Ground Water, (2011), 49 (3), 319-323.

[6] J. Lions, R. Teman, S. Wang. On the equations of the large-scale ocean. Nonlinearity, (1992), 5, $1007-1053$.

[7] J. Lions, R. Teman, S. Wang. New formulations of the primitive equations of atmosphere and applications. Nonlinearity, (1992), 5, 237-288.

[8] J. McCreary. Easern tropical ocean response to changing wind systems with applications to El Niño. J. Phys, Oceanogr., (1976), 6, 632-645.

[9] J. McCreary. A linear stratified ocean model of the equatorial undercurrent. Phil. Trans. Roy. Soc. London., (1981), 302, 385-413.

[10] J. McCreary. E quatorial beams. J. Mar. Res., (1984), 42, 395-430.

[11] P. Müller, G. Holloway, F. Henyey, N. Pomphrey. Nonlinear interactions among internal gravity waves. Rev. Geophys., (1986), 24, 3, 493-536.

[12] D. Nethery, D. Shankar. Vertical propagation of baroclinic Kelvin waves along the west coast of India. J. Earth. Syst. Sci., (2007), 116 (4), 331-339.

[13] R. Romea, J. Allen. On vertically propagating coastal Kelvin waves at low latitudes. J. Phys. Oceanogr., (1983), 13 (1),241-1,254.

[14] D. Shindell, G. Schmidt. Southern Hemisphere climate response to ozone changes and greenhouse gas increases. Res. Lett., (2004), 31, L18209. 
[15] C. Staquet, J. Sommeria. Internal Gravity Waves: From instabilities to turbulence. Annu. Rev. Fluid Mech., (2002), $34,559-593$.

[16] C. Summerhayes, S. Thorpe. Oceanography, An Illustrative Guide, New York: John Willey ES Sons, (1996).

[17] R. Szoeke, R. Samelson. The duality between the Boussinesq and non-Boussinesq hydrostatic equations of motion. J. Phys. Oceanogr., (2002), 32, 2194-2203.

[18] A. Timmermann, M. Latif, A. Grotzner, R. Voss, R., Modes of climate variability as simulated by a copled general circulation model. Part I: ENSO-like climate variability and its low-frequency modulation. Climate Dynamics., (1999), 15 (8), 605-618.

[19] G. Watson. A Treatise on the Theory of Bessel Functions, Second edition, Cambridge University Press, (1996)(ISBN13: 9780521483919 - ISBN-10: 0521483913). 